\title{
Biolubrifiants et écolabels
}

François VAN DIEVOET

BfB Oil Research S.A., Parc scientifique Crealys,

rue Phocas Lejeune 10,

B-5032 Gembloux.

Fax: +3281585308

<francois.vandievoet@bfblab.com>

La quantité de biolubrifiants utilisée en France et en Europe est aujourd'hui homéopathique. La régression est importante puisque 4000 ans avant JC ils couvraient $100 \%$ du marché : ils ne représentent plus que 0,01\% aujourd'hui.

Il y a à cela un certain nombre de raisons, dont la première à mon avis réside au niveau de la qualité. II faut reconnaître que dans un certain nombre de domaines les lubrifiants élaborés sur base renouvelables, essentiellement végétale, ne sont pas du niveau des produits d'origine pétrolière. Vraisemblablement en raison de travaux encore insuffisants de recherche et développement. Ce retard est en train d'être rattrapé et les esters élaborés sur base de tournesol, colza et autres matières premières végétales croissent rapidement en qualité et devraient bientôt permettre de surmonter un certains nombre de problèmes comme celui de la dénitrification des huiles moteurs avec des matières renouvelables. Ce qui représente évidemment un challenge très important puisque ce domaine concerne $50 \%$ de la consommation de lubrifiants dans le monde.

Vraisemblablement aussi, au début, ce sont de petites entreprises qui se sont occupées à formuler des produits que l'on appelle « bio » et souvent la qualité n'était pas au rendez-vous par manque de moyens techniques et de connaissances. Ce qui fait que ces produits n'ont pas donné une image de marque valorisante aux produits d'origine végétale.

La deuxième raison est d'ordre économique. On est aidé aujourd'hui par l'augmentation très importante du prix des produits pétroliers. Une situation qui $n^{\prime}$ est pas près de disparaître car il devient de plus en plus évident que dans un laps de temps assez court, quelques décennies, on sera confronté à la fin du pétrole. Comme dirait Salvador Dali, tout cela à l'échelle cosmique n'est qu'un détail, mais c'est un détail important pour nous. Donc il est temps de prendre déjà les mesures pour essayer de remplacer un certain nombre de

Abstract: Presentation of european ecolabels for lubricants: the ecolabel of CEE, blue angel, white swan.

Key words: Europe, ecolabel, biodegradability, ecotoxicity

produits pétroliers dans certaines applications, par d'autres produits.

La troisième raison qui me vient à l'esprit quand je pense au peu de pénétration des produits " bio ", - j'entends par là des produits provenant de la biomasse (et non nécessairement biodégradables) - c'est effectivement le manque de transparence sur ce qu'est un produit bio.

Mettez-vous à la place du consommateur.

Il était donc nécessaire de mettre en place des écolabels et ils portent aujourd'hui sur tout un ensemble de produits les peintures, les papiers, les machines à laver, et même les ordinateurs.

L'Allemagne fut le premier pays à adopter cette démarche et a créé sur les traces d'une réglementation sur la protection des eaux, le fameux «Ange bleu » qui peut être considéré comme le précurseur de tous les écolabels qui ont suivi. Le Japon, le Canada, la Thaillande, les Pays scandinaves avec le « Cygne blanc», ont chacun à leur tour défini leur écolabel.

Si bien qu'il est apparu nécessaire de définir un écolabel européen. Ainsi, il y a trois ans, un groupe de travail s'est réuni constitué de représentants de l'industrie des lubrifiants, de producteurs de matériel de représentants des ministères de l'environnement de l'Europe des 15. Une structure hollandaise, Ivam, relevant de I'université d'Amsterdam, a été chargée de la coordination des travaux. Et en janvier 2005, le travail final a été approuvé par les pays de I'Union (devenus 25) plus deux (la Norvège et la Suisse).

Depuis janvier 2005, l'Europe dispose enfin d'un écolabel consacré aux lubrifiants.

Dans un premier temps, il est apparu inapproprié d'étendre cette spécification à tous les lubrifiants et il a été ainsi décidé de se concentrer sur les produits les plus importants aujourd'hui du point de vue de leur impact immédiat sur l'environnement.

Cet écolabel, dans un premier temps, s'adresse ainsi aux lubrifiants suivants :
- les fluides hydrauliques (utilisés dans les travaux publics, les travaux forestiers, l'agriculture, etc.) :

- les graisses ;

- les huiles de chaîne de tronçonneuse et autres lubrifiants à usage perdu (ex : huiles de démoulage);

- les huiles moteurs 2-temps (dans le cadre des deux temps marins où la combustion de I'huile et du carburant via l'échappement se retrouve dans l'eau).

L'écolabel européen fixe non seulement les critères environnementaux mais aussi les exigences techniques que doivent satisfaire les lubrifiants (le niveau de qualité des produits). D'autre part, il oblige, pour chacun de ces lubrifiants, à une utilisation minimale d'huile de base d'origine renouvelable : cette notion supplémentaire n'existe pas dans «l'Ange Bleu ». Nous parlerons tour à tour de :

- I'Ecolabel CEE,

- I'Ange Bleu,

- le Nordic Swan,

- la norme NF Environnement,

- des exigences techniques existantes pour les écolubes,

- I'Approche US pour EFL.

\section{L'écolabel CEE}

Sept critères définissent les spécifications des écolubrifiants.

Ils doivent présenter une validité de 4 ans.

Le dossier technique déposé devant l'autorité compétente (pour la France, l'Afnor) doit présenter des données provenant de laboratoires accrédités 17025.

Critère 1 : le produit ne peut présenter de $R$ phrases indiquant un danger pour l'environnement et pour la santé selon la directive européenne sur les préparations dangereuses, en particulier la série 50,51,52,53 qui porte sur les critères amenant à qualifier le produit de dangereux pour l'environnement. 
Tableau 1. Exigences de toxicité aquatique pour les différents sous-groupes de lubrifiants.

\begin{tabular}{|c|c|c|c|c|}
\hline Critère 2.1. & Fluides hydrauliques & Graisse* & $\begin{array}{l}\text { Huiles de chaînes et autres } \\
\text { lubrifiants à usage perdu }\end{array}$ & Huiles 2 temps \\
\hline $\begin{array}{l}\text { Toxicité aquatique pour produit } \\
\text { complètement formulé dans les } 3 \text { tests } \\
\text { OECD : } 201 \text { - } 202 \text { - } 203\end{array}$ & $\geq 100 \mathrm{mg} / \mathrm{L}$ & $\geq 1000 \mathrm{mg} / \mathrm{L}$ & $\geq 1000 \mathrm{mg} / \mathrm{L}$ & $\geq 1000 \mathrm{mg} / \mathrm{L}$ \\
\hline $\begin{array}{l}\text { Toxicité aquatique pour chaque } \\
\text { composant principal dans les tests : } \\
201 \text { et } 202\end{array}$ & $\geq 100 \mathrm{mg} / \mathrm{L}$ & $\geq 100 \mathrm{mg} / \mathrm{L}$ & $\leq 100 \mathrm{mg} / \mathrm{L}$ & $\geq 100 \mathrm{mg} / \mathrm{L}$ \\
\hline
\end{tabular}

* Les graisses peuvent être évaluées de cette manière si l'épaississant montre une biodégradation > 70 \% dans OECD 302.

Le produit doit être accompagné d'une fiche de données sécurité, établie selon les directives de la Commission 1991/155EEC et 675/48EEC, et chacun de ses composants doit faire l'objet d'un enregistrement EINECS ou ELINCS (notification aujourd'hui en vigueur et qui sera bientôt remplacée par Reach).

Le critère 2 présente les exigences pour les composants principaux (tableau 1). Dans le cadre de l'écolabel, on regarde non seulement le produit final au regard de ses performances vis-à-vis de l'environnement, mais également les performances de chacun des composants. Les contraintes sont différentes selon le lubrifiant concerné (fluides hydrauliques, graisses, huiles de chaînes et autres lubrifiants à usage perdu, huiles 2-temps) et portent sur la toxicité aquatique pour la formulation complète (tests OECD : 201-202-203 : algues, les daphnies et les poissons) et pour chaque composant (tests OCED 201 et 202). Ce critère permet l'usage d'un certain nombre de produits dangereux ou toxiques en fixant un seuil-limite de concentration (tableau 2).

Le critère 3 a trait au potentiel de biodégradabilité et de bioaccumulation (tableau 3). Pour chaque catégorie d'écolubrifiant est autorisé un certain pourcentage de produits non biodégradables.
Un produit biodégradable est un produit qui dans la série d'essais 301 obtient un résultat supérieur à $70 \%$. Si ce produit atteint un pourcentage de $30 \%$ on peut éventuellement lui faire subir un test de biodégradabilité intrinsèque (test de la série 302). Ce test donne une idée du potentiel de biodégradabilité : on mesure ici la biodégradabilité du carbone (et non des autres produits). Et on considère qu'un produit présente un potentiel de biodégradabilité si son résultat est supérieur à 20 : cette situation concerne par exemple $90 \%$ des additifs utilisés dans les lubrifiants. II n'est pas obligatoire mais utile de connaître le taux de bioaccumulation : ce

Tableau 2. Exigences de toxicité aquatique pour chaque sous-groupe de produits. Exigences en matière de données concernant chaque composant.

\begin{tabular}{|c|c|c|c|c|}
\hline \multirow{2}{*}{$\begin{array}{l}\text { Critère } 2.2 \\
\text { Toxicité aquatique }\end{array}$} & \multicolumn{4}{|c|}{ Concentration en masse cumulée des substances présentes dans } \\
\hline & $\begin{array}{c}\text { Fluides } \\
\text { hydrauliques }\end{array}$ & Graisses & $\begin{array}{l}\text { Huiles pour scies à chaînes, } \\
\text { agents de décoffrage du béton } \\
\text { et autres produits de graissage d'appoint }\end{array}$ & $\begin{array}{l}\text { Huiles pour moteur } \\
\text { à deux temps }\end{array}$ \\
\hline $\begin{array}{l}10 \mathrm{mg} / \mathrm{L}<\text { toxicité aiguë } \leq 100 \mathrm{mg} / \mathrm{L} \\
\text { ou } 1 \mathrm{mg} / \mathrm{L}<\text { CSEO } \leq 10 \mathrm{mg} / \mathrm{L}\end{array}$ & $\leq 20$ & $\leq 25$ & $\leq 5$ & $<25$ \\
\hline $\begin{array}{l}1 \mathrm{mg} / \mathrm{L}<\text { toxicité aiguë* } \leq 10 \mathrm{mg} / \mathrm{L} \\
\text { ou } 0,1 \mathrm{mg} / \mathrm{L} \text { CSEO } \leq 1 \mathrm{mg} / \mathrm{L}\end{array}$ & $\leq 5$ & $1 \leq$ & $\leq 0,5$ & $\leq 1$ \\
\hline $\begin{array}{l}\text { Toxicité aiguë̈* }<1 \mathrm{mg} / \mathrm{L} \\
\text { ou CSEO } \leq 0,1 \mathrm{mg} / \mathrm{L}\end{array}$ & $\leq 1$ & $\leq 0,1$ & $\leq 0,1$ & $\leq 0,1$ \\
\hline
\end{tabular}

* CE50/CL50/Cl50

Table 3. Critère 3 : potentiel de biodégradabilité et bioaccumulation.

\begin{tabular}{|c|c|c|c|c|}
\hline \multirow[b]{2}{*}{ Biodégradation } & \multicolumn{4}{|c|}{ Masses cumulées contenues dans les substances } \\
\hline & Fluides hydrauliques & Graisses & $\begin{array}{l}\text { Huiles de chaines, huiles de décoffrages } \\
\text { et autres lubrifiants à usage perdu }\end{array}$ & Huiles 2-temps \\
\hline Non-biodégradabilité* & $\leq 5$ & $\leq 10$ & $\leq 5$ & $\leq 10$ \\
\hline $\begin{array}{l}\text { Biodégradabilité aérobie } \\
\text { intrinsèque }\end{array}$ & $\leq 5$ & $\leq 20$ & $\leq 5$ & $\leq 20$ \\
\hline Biodégradabilité aérobie ultime & $>90$ & $>75$ & $>90$ & $>75$ \\
\hline
\end{tabular}

* Les substances non biodégradables sont autorisées dans le lubrifiants jusqu'à certains pourcentages pour autant qu'elles ne soient pas en même temps bioaccumulatives. En d'autres mots, les substances non biodégradables ni bioaccumulatives ne devraient pas être ajoutées au lubrifiants.

Méthodes de tests

Biodégradabilités aérobie intrinsèque : OECD $302 \mathrm{C}(>70 \%)$ ou $>20 \%$ mais $<60 \%$ dans OECD $301 \mathrm{~B}$.

Biodégradabilité aérobie ultime : OECD $301 \mathrm{~B}(\geq 60 \%)$.

Bioaccumulation selon OECD 117 . 
taux peut se mesurer de deux façons soit par calcul du coefficient de partage dans l'eau, ou par mesure de l'accumulation dans le poisson (on mesure alors la quantité de produits accumulés : des métaux lourds ou des molécules que le poisson ne peut utiliser comme matière nutritive, ni rejeter).

Le critère exclut un certain nombre de substances : il reprend pour cela la liste prioritaire de I'EU pour la politique de l'eau, la liste OSPAR (Oslo-Paris) - une liste de produits chimiques (version de décembre 2004) mise en place par la France, la Norvège et l'Angleterre et qui définit les produits à proscrire sur les plateformes de forages en Mer du Nord afin de protéger les milieux marins; sont également interdits les composants organiques halogènes, les nitrites et les métaux (non intentionnellement ajoutés) sauf $\mathrm{Na}, \mathrm{K}, \mathrm{Mg}, \mathrm{Ca}$, Li, Al.

Le critère 5 (introduit sur la pression des pays scandinaves) fixe les teneurs minimales en carbone d'origine renouvelable selon les produits :

$-\geq 50 \%$ huiles hydrauliques ;

$-\geq 70$ \% huiles de chaînes, agents de démoulage et de décoffrage et autres lubrifiants à usage perdu ;

$-\geq 45 \%$ graisses;

$-\geq 50 \%$ huiles 2 temps.

Le critère 6 définit les performances techniques du produit. II y a peu de spécifications techniques spécifiques aux produits " bio ». Ce sont deux normes internationales (ISO 15380 pour huiles hydrauliques).

ANGE BLEU RAL-UZ-48 pour huiles de chaînes. NMMA TC-W3 pour lubrifiants pour moteurs à essence 2-temps.

Adapter pour tout autre lubrifiant à usage perdu.

Le dernier critère 7 oblige à faire apparaître sur l'emballage du produit l'allégation suivante : « Dommage réduit pour l'eau et le sol durant I'utilisation ; émissions de $\mathrm{CO}_{2}$ réduites ».

\section{L'Ange bleu}

Maintenant que nous disposons d'un écolabel européen, les différents écolabels nationaux devraient être amenés à céder la place.

Ainsi, pour l'Ange bleu : la révision prévue en 2005 n'a pas eu lieu et vraisemblablement la réglementation allemande s'orientera vers la demande de produits satisfaisant l'écolabel européen.

Les différences de l'Ange bleu (tableau 4) avec l'écolabel européen portent en particulier sur la présence d'un test d'écotoxicité sur les plantes (qui n'existe pas dans l'écolabel européen) et pour les additifs la présence d'un essai de toxicité sur les bactéries.

Table 4. Ange bleu.

\begin{tabular}{|ll|}
\hline Substances de base & \multicolumn{1}{c|}{ Additifs } \\
\hline Biodégradabilité & Biodégradabilité \\
$>70 \%$ OECD $301 \mathrm{~A} / \mathrm{E} / \mathrm{B} / \mathrm{F} / \mathrm{D} / \mathrm{C}$ & OECD $301 \mathrm{~A} / \mathrm{E} / \mathrm{B} / \mathrm{F} / \mathrm{D} / \mathrm{C}$ \\
Si toxicité supposée $<10 \mathrm{mg} / \mathrm{L}$ & Si toxicité supposée $\mathrm{EC}_{50} \leq 1 \mathrm{mg} / \mathrm{L}$ \\
& Ou toxicité $\mathrm{EC}_{30} \leq 100 \mathrm{mg} / \mathrm{L}+$ bioaccumulable (log Pow $\left.\geq 3.0\right)$ \\
Toxicité & Toxicité \\
Daphanies OECD 202 & Daphanies OECD 202 \\
Poissons OECD 203 & Poissons OECD 203 \\
Algues OECD 201 & Algues OECD 201 \\
Plantes OECD 208 & Inhibition des bactéries \\
& Plantes OECD 208 \\
& Si toxicités $>$ mg/L $\rightarrow$ Ange bleu \\
\hline
\end{tabular}

\section{Le Cygne blanc}

Le Cygne blanc - très inspiré de l'Ange bleu - exige la présence de produits d'origine renouvelables.

Son application s'étend à un plus grand nombre de produits que l'écolabel européen mais cette extension fera l'objet des étapes suivantes du développement de ce dernier (tableau 5).

\section{NF environnement}

Cette norme est très proche de ce qu'est aujourd'hui l'écolabel européen.

\section{L'approche de I'ASTM (États-Unis)}

Les États-Unis s'intéressent depuis une quinzaine d'années à ces produits.

L'ASTM (organisation équivalente de l'Afnor en France) a mis en place un certain nombre de spécifications de méthodes d'essais.

Par exemple, les tests de mesure de la biodégradabilité (qui parfois sont la copie exacte des tests européens) :

- D 5864 - Biodégradabilité (sturm),

- D 6139 - Biodégradabilité (gledhill flash),

-D 6731 - Biodégradabilité (respiromètre fermé).

Table 5. Cygne blanc.

\begin{tabular}{|lc|}
\hline Huile de base & \multicolumn{1}{c|}{ Additifs } \\
\hline Biodégradagilité & Biodégradagilité \\
OECD $301 \mathrm{~B}$ ou $\mathrm{F}$ (iso 9439) & OECD $301 \mathrm{~B}$ ou F (iso 9439) \\
& Facilement biodégradable \\
Toxicité & Toxicité \\
$\begin{array}{l}\text { Daphanies OECD } 202 \rightarrow>100 \mathrm{mg} / \mathrm{l} \\
\text { Algues OECD } 201 \rightarrow>100 \mathrm{mg} / \mathrm{l}\end{array}$ & Daphanies OECD $202 \rightarrow>100 \mathrm{mg} / \mathrm{l}$ \\
& Algues OECD $201 \rightarrow>100 \mathrm{mg} / \mathrm{l}$ \\
& Bioaccumulation : log Pow $<3$ \\
& OECD 107/117 \\
\hline
\end{tabular}

Doit satisfaire aux exigences physico-chimiques et aux performances
Ils ont également défini des tests pour la

Quel est le marché des lubrifiants biodégradables aux USA? Dans le 2 temps, $70 \%$ des lubrifiants sont biodégradables. Dans d'autres domaines, on voit également des percées des biolubrifiants: par exemple dans les Parcs nationaux américains il est strictement obligatoire d'utiliser des biolubrifiants.

\section{Revue des spécifications techniques définissant les critères physico-chimiques et de performances requis pour obtenir l'écolabel}

Les produits labélisables.

On peut considérer comme labélisables :

- les huiles pour moteurs hors bord 2-temps;

- les lubrifiants pour chaînes ;

- les lubrifiants pour le démoulage ;

- les hydrauliques (mobile);

- tout ce qui est proche du traitement de l'eau (sites de vidange, turbines, écluses) ; produits utilisés dans la sidérurgie dont $100 \%$ sont d'origine pétrolière) ;

- les engrenages ouverts;

- le travail des métaux ; mesure de l'écotoxicité.

- les produits anti-corrosion (très nombreux 
- les usages liés aux voies ferrées (roues, aiguillages des voies, etc.)

\section{Conclusion}

La mise à disposition des autorités d'un écolabel concernant les lubrifiants les plus sensibles pour protéger l'environnement devrait permettre la mise en place de règles et de lois obligeant I'utilisation de ce type de lubrifiants Un des verrous qui empêchait le développement des biolubrifiants est levé.

Le contexte des prix a changé et nous est devenu plus favorable.
Par ailleurs, l'industrie productrice de lubrifiants possède dans sa gamme les produits adéquats. Encore faut-il inciter les consommateurs à en faire usage dans l'intérêt de tous.

La balle est désormais de leur côté. 